\title{
High PKM2 expression is independently correlated with decreased overall survival in hepatocellular carcinoma
}

\author{
DONG-HUI LU ${ }^{1 *}$, WEN-WEN LV ${ }^{2,3^{*}}$, WEN-XING LI ${ }^{4,5}$ and YUE-DONG GAO ${ }^{4,6}$ \\ ${ }^{1}$ Department of Oncology, People's Liberation Army 105 Hospital, Hefei, Anhui 230031; ${ }^{2}$ Hongqiao International Institute of \\ Medicine, Shanghai Tongren Hospital/Faculty of Public Health; ${ }^{3}$ Clinical Research Center, Shanghai Jiao Tong University \\ School of Medicine, Shanghai 200025; ${ }^{4}$ Key Laboratory of Animal Models and Human Disease Mechanisms, Kunming \\ Institute of Zoology, Chinese Academy of Sciences, Kunming, Yunnan 650223; ${ }^{5}$ Kunming College of Life Science, \\ University of Chinese Academy of Sciences, Kunming, Yunnan 650204; ${ }^{6}$ Kunming Biological Diversity Regional \\ Center of Instruments, Kunming Institute of Zoology, Chinese Academy of Sciences, \\ Kunming, Yunnan 650223, P.R. China
}

Received January 23, 2018; Accepted July 20, 2018

DOI: $10.3892 / \mathrm{ol} .2018 .9100$

\begin{abstract}
Hepatocellular carcinoma (HCC) is one of the most lethal and malignant types of cancer that affects global human health. The present study aimed to investigate the effect of pyruvate kinase muscle isozyme M2 (PKM2) expression on the clinical features and prognosis of HCC. The present study employed univariate logistic regression to investigate the correlation between PKM2 expression and clinical features. Univariate and multivariate Cox regression analyses were performed to estimate the independent effect of PKM2 expression on survival status. The results revealed that patients in the high PKM2 group $(\geq 11.25)$ exhibited significantly lower creatinine levels $(\mathrm{P}=0.043)$, higher fetoprotein levels $(\mathrm{P}<0.001)$, advanced stage $(\mathrm{P}<0.001)$ and higher grade $(\mathrm{P}=0.004)$ compared with patients with low PKM2 expression levels $(<11.25)$. In addition, patients with high PKM2 expression exhibited poor prognosis compared with patients with low PKM2 expression. After correcting the covariates, PKM2 expression remains significantly associated with
\end{abstract}

Correspondence to: Dr Wen-Xing Li, Key Laboratory of Animal Models and Human Disease Mechanisms, Kunming Institute of Zoology, Chinese Academy of Sciences, 32 Jiaochang Donglu, Kunming, Yunnan 650223, P.R. China

E-mail: liwenxing2016@gmail.com

Dr Yue-Dong Gao, Kunming Biological Diversity Regional Center of Instruments, Kunming Institute of Zoology, Chinese Academy of Sciences, 32 Jiaochang Donglu, Kunming, Yunnan 650223, P.R. China

E-mail: gaoyd@mail.kiz.ac.cn

*Contributed equally

Key words: pyruvate kinase muscle isozyme M2, hepatocellular carcinoma, clinical features, overall survival reduced overall survival $(\mathrm{P}<0.05)$. These findings suggested that PKM2 is an independent risk factor for $\mathrm{HCC}$ and provides valuable information for future studies on the pathogenesis of HCC and drug discovery.

\section{Introduction}

Hepatocellular carcinoma (HCC) is a major type of liver cancer and accounts for $85-90 \%$ of all primary liver cancers (1). HCC is the sixth most common type of cancer as well as the third most frequent cause of cancer-related deaths (2). It is more prevalent and deadly in developing countries $(3,4)$. China has the highest rate of $\mathrm{HCC}$ worldwide, which accounts for $55 \%$ of all cases (5). The incidence rate for liver cancer in Chinese men and women is 38.32 and 13.85 per 100,000 , respectively (6).

It is widely accepted that hepatocarcinogenesis is a long-term process involving a series of genetic and epigenetic alterations that take place in multiple steps, including initiation, promotion, malignant conversion and progression of disease (7). Furthermore, a recent study showed that the 5-year survival rate was $57.0 \%$ and the median survival time was 60 months in HCC patients after curative resection (8). The prognosis of $\mathrm{HCC}$ is still quite poor as a result of high recurrence rates, which might be attributed to the lack of reliable molecular markers that reflect tumor behavior, such as tumor growth and progression $(9,10)$. Therefore, efforts have being made to reveal the complex molecular mechanisms underlying the initiation, propagation and progression of HCC for decades (7). Previous studies identified a large number of genes, proteins and other molecules that are associated with diverse cellular processes and pathways that are involved in cell cycle, the JAK/STAT signaling pathway, the Wnt/ $\beta$-catenin signaling pathway, the p53 signaling pathway and Ras signaling (11-14).

Pyruvate kinase muscle isozyme M2 (PKM2) is one of the isoforms of pyruvate kinase $(\mathrm{PK})$ that is responsible for the final rate-limiting step in catalyzing glycolysis and is universally expressed during embryogenesis, tissue regeneration, tumor development and normal proliferating 
cells $(13,15,16)$. PKM2 also has a major role in the dysregulated metabolism programs in other types of proliferating cells and cancer cells (17-19). PKM2 is over-expressed in a broad range of human cancers (20-24). Moreover, PKM2 has recently generated the greatest interest out of all PK isoforms as it not only have a huge effect on cellular metabolism in cancer but also immunometabolism (25).

In our previous study, we observed the positive expression rate of PKM2 to be $53.5 \%$ in liver tumor tissues and $27.1 \%$ in adjacent tissues in the Chinese population (26). The expression of PKM2 in tumor tissues was significantly higher than in adjacent non-tumor liver tissues (26). Although there have been many studies that previously reported on the correlation between PKM2 and HCC prognosis (27-29), whether PKM2 independently affects the prognosis of HCC is still not clear as prognosis may be affected by multiple confounding factors.

Therefore, in the present study, we aimed to investigate the independent effect of high PKM2 expression on the prognosis of HCC patients and eliminate other confounding factors using liver hepatocellular carcinoma (LIHC) RNA-seq and clinical data from The Cancer Genome Atlas (TCGA) database. The present study will provide stronger evidence for the detection of PKM2 mRNA and protein expression in liver cancer cell lines in our future investigations.

\section{Materials and methods}

Data collection. RNA-seq expression and clinical data on LIHC were downloaded from the Cancer Genome Atlas (TCGA, https://tcga-data.nci.nih.gov/) and cBioPortal database (30). We downloaded TCGA level 3 data, including data from 371 primary HCC patients. All samples were tested by Illumina HiSeq 2000 RNA Sequencing v.2 analysis. RSEM (RNA-Seq by Expectation-Maximization) expression values were used for statistical analysis.

Samples grouping. We divided the liver cancer samples into two groups according to the logarithmic converted median expression value of PKM2 (11.25). Case samples with PKM2 expression $\geq 11.25$ were considered as the high-PKM2 group, and those with low expression of PKM2 $(<11.25)$ as the low-PKM2 group; variables in these two groups were compared using Student's t-tests, and the prevalence of categorical variables was compared using $\chi^{2}$ tests.

Statistical analysis. Univariate logistic regression was used to investigate the correlation between PKM2 expression (as a categorical dependent variable) and clinical features. Differences in overall survival and disease-free survival between high PMK2 expression and low PKM2 expression groups were compared using Kaplan-Meier curves with P-values calculated via the log-rank test using the Survival package in $\mathrm{R}$. Univariate Cox regression analysis was used to estimate the independent effect of PKM2 expression and other clinicopathological factors (sex, diagnosis age, BMI, creatinine, fetoprotein, albumin, residual tumor, vascular tumor invasion, adjacent hepatic tissue inflammation, stage and grade) on overall survival and disease-free survival. Multivariate Cox analysis was used to compare the effect of PKM2 expression on overall survival and disease-free survival. The corresponding covariates were adjusted.

\section{Results}

Patient characteristics. The characteristics of 371 primary tumor patients stratified by median PKM2 expression (11.25) are presented in Table I. There were 186 case samples in the high PKM2 expression group and 185 case samples in the low PKM2 expression group. There were more males who exhibited low PKM2 expression, and females exhibited higher PKM2 expression $(\mathrm{P}<0.001)$. There were no differences in age at diagnosis, height, weight, BMI, albumin level, residual tumor, vascular tumor invasion or adjacent hepatic tissue inflammation between the high PKM2 expression and low PKM2 expression groups. However, patients in the high PKM2 expression group showed lower creatinine levels $(\mathrm{P}=0.043)$ and higher fetoprotein levels $(\mathrm{P}<0.001)$ compared with the low PKM2 expression group. In addition, more patients at stage III/IV or grade $3 / 4$ were in the high PKM2 expression group compared with the low PKM2 expression group $(\mathrm{P}<0.001$ and $\mathrm{P}=0.004$, respectively).

PKM2 expression and clinicopathological characteristics. PKM2 expression was correlated with creatinine, fetoprotein, pathologic stage and neoplasm histologic grade (Table I). The boxplot of these four variables and PKM2 expression are displayed in Fig. 1. With the exacerbation of pathologic stage, PKM2 expression was increased (Fig. 1C). We also observed the same trend in the correlation between neoplasm histologic grade and PKM2 expression (Fig. 1D). In addition, the Kaplan-Meier curves showed that a high PKM2 expression was related to poor overall survival and disease-free survival (Fig. 1E and F). We further analyzed PKM2 expression and survival status data that were stratified by stage and grade, and found that a high PKM2 expression resulted in poor overall and disease-free survival in patients with grade 1 or 2 (data not shown).

We used unconditional logistic regression model to investigate the associations between PKM2 expression and clinicopathological variables (Table II). Female sex (OR=2.407, $\mathrm{P}<0.001)$, high fetoprotein level $(\mathrm{OR}=1.157, \mathrm{P}<0.001), \mathrm{R} 1$ or $\mathrm{R} 2$ residual tumor $(\mathrm{OR}=2.293, \mathrm{P}=0.020)$, high pathologic stage $(\mathrm{OR}=1.857, \mathrm{P}=0.013)$ and high neoplasm histologic grade $(\mathrm{OR}=2.010, \mathrm{P}=0.002)$ were positively correlated with $\mathrm{PKM} 2$ expression.

PKM2 expression, clinicopathological variables and patient survival. In the univariate Cox regression model, we found a high PKM2 expression (both continuous and categorical) was significantly associated with lower overall survival $(H R=1.192$, $\mathrm{P}=0.001$ for continuous data and $\mathrm{HR}=1.740, \mathrm{P}=0.002$ for categorical data, respectively; Table III) as well as lower disease-free survival $(\mathrm{HR}=1.129, \mathrm{P}=0.016$ for continuous data and $\mathrm{HR}=1.406, \mathrm{P}=0.025$ for categorical data, respectively; (Table IV). In addition, $\mathrm{R} 1$ or $\mathrm{R} 2$ residual tumor $(\mathrm{HR}=2.033$, $\mathrm{P}=0.007)$ and high stage $(\mathrm{HR}=2.443, \mathrm{P}<0.001)$ also caused poor overall survival. Furthermore, high fetoprotein levels $(\mathrm{HR}=1.038, \mathrm{P}=0.047), \mathrm{R} 1$ or $\mathrm{R} 2$ residual tumor $(\mathrm{HR}=1.763$, $\mathrm{P}=0.021)$, micro or macro vascular tumor invasion $(\mathrm{H}=1.990$, 
Table I. Hepatocellular carcinoma patients' characteristics stratified by PKM2 expression.

\begin{tabular}{|c|c|c|c|}
\hline \multirow[b]{2}{*}{ Patient characteristics } & \multicolumn{2}{|c|}{ PKM2 expression } & \multirow[b]{2}{*}{ P-value } \\
\hline & High $(\geq 11.25)$ & Low $(<11.25)$ & \\
\hline Sex, n (\%) & & & $<0.001$ \\
\hline Male & $107(57.8)$ & $142(76.8)$ & \\
\hline Female & $78(42.2)$ & $43(23.2)$ & \\
\hline Diagnosis age, years & $58.4 \pm 14.3$ & $60.5 \pm 12.7$ & 0.129 \\
\hline Height, $\mathrm{cm}$ & $166.7 \pm 9.6$ & $168.6 \pm 8.6$ & 0.057 \\
\hline Weight, kg & $71 \pm 20.2$ & $74.6 \pm 18.6$ & 0.087 \\
\hline Body mass index, $\mathrm{kg} / \mathrm{m}^{2}$ & $25.5 \pm 6.8$ & $26.1 \pm 5.5$ & 0.397 \\
\hline Creatinine, $\mathrm{mg} / \mathrm{dl}$ & $1.1 \pm 1.0$ & $2.8 \pm 10.9$ & 0.043 \\
\hline Fetoprotein, ng/ml (log2 converted) & $6.8 \pm 5.0$ & $4.1 \pm 3.4$ & $<0.001$ \\
\hline Albumin, g/dl & $3.9 \pm 0.9$ & $4.4 \pm 5.6$ & 0.149 \\
\hline Race, n (\%) & & & 0.973 \\
\hline Asian & $76(42.5)$ & $81(44.8)$ & \\
\hline White & $93(52.0)$ & $91(50.3)$ & \\
\hline Black/African American & $9(5.0)$ & $8(4.4)$ & \\
\hline American Indian/Alaska Native & $1(0.6)$ & $1(0.6)$ & \\
\hline Residual tumor, n (\%) & & & 0.181 \\
\hline R0 & $154(92.8)$ & $170(96.6)$ & \\
\hline $\mathrm{R} 1 / \mathrm{R} 2$ & $12(7.2)$ & $6(3.4)$ & \\
\hline Vascular tumor invasion & & & 0.094 \\
\hline None & $90(60.4)$ & $116(69.9)$ & \\
\hline Micro & $48(32.2)$ & $45(27.1)$ & \\
\hline Macro & $11(7.4)$ & $5(3)$ & \\
\hline Adjacent hepatic tissue inflammation & & & 0.268 \\
\hline None & $53(50.0)$ & $64(50.0)$ & \\
\hline Mild & $48(45.3)$ & $51(39.8)$ & \\
\hline Severe & $5(4.7)$ & $13(10.2)$ & \\
\hline Stage, n (\%) & & & $<0.001$ \\
\hline I & $65(38)$ & $106(59.9)$ & \\
\hline II & $51(29.8)$ & $35(19.8)$ & \\
\hline III & $50(29.2)$ & $35(19.8)$ & \\
\hline IV & $5(2.9)$ & $1(0.6)$ & \\
\hline Grade, n (\%) & & & 0.004 \\
\hline 1 & $19(10.3)$ & $36(19.8)$ & \\
\hline 2 & $83(45.1)$ & 94 (51.6) & \\
\hline 3 & $76(41.3)$ & $46(25.3)$ & \\
\hline 4 & $6(3.3)$ & $6(3.3)$ & \\
\hline
\end{tabular}

PKM2, pyruvate kinase muscle isozyme M2.

$\mathrm{P}<0.001)$ and high stage $(\mathrm{HR}=2.376, \mathrm{P}<0.001)$ were correlated with poor disease-free survival. Based on these results, we adopted multivariate Cox analysis to investigate the associations between PKM2 expression and overall survival with the adjustment of residual tumor and stage. Both continuous and categorical data on PKM2 expression were significantly correlated with overall survival $(\mathrm{HR}=1.119, \mathrm{P}=0.050$ for continuous and $\mathrm{HR}=1.477, \mathrm{P}=0.042$ for categorical, respectively; Table V). However, the results of the analysis of the association between PKM2 expression levels and disease-free survival were not significant with the adjustment of fetoprotein, residual tumor, vascular tumor invasion and stage (Table V). These results suggested that a high expression of PKM2 was independently associated with overall survival in HCC patients.

\section{Discussion}

HCC is frequently diagnosed at advanced stages. It is a highly complex and devastating disease with limited and largely ineffective therapeutic options $(31,32)$. Consequently, considerable 

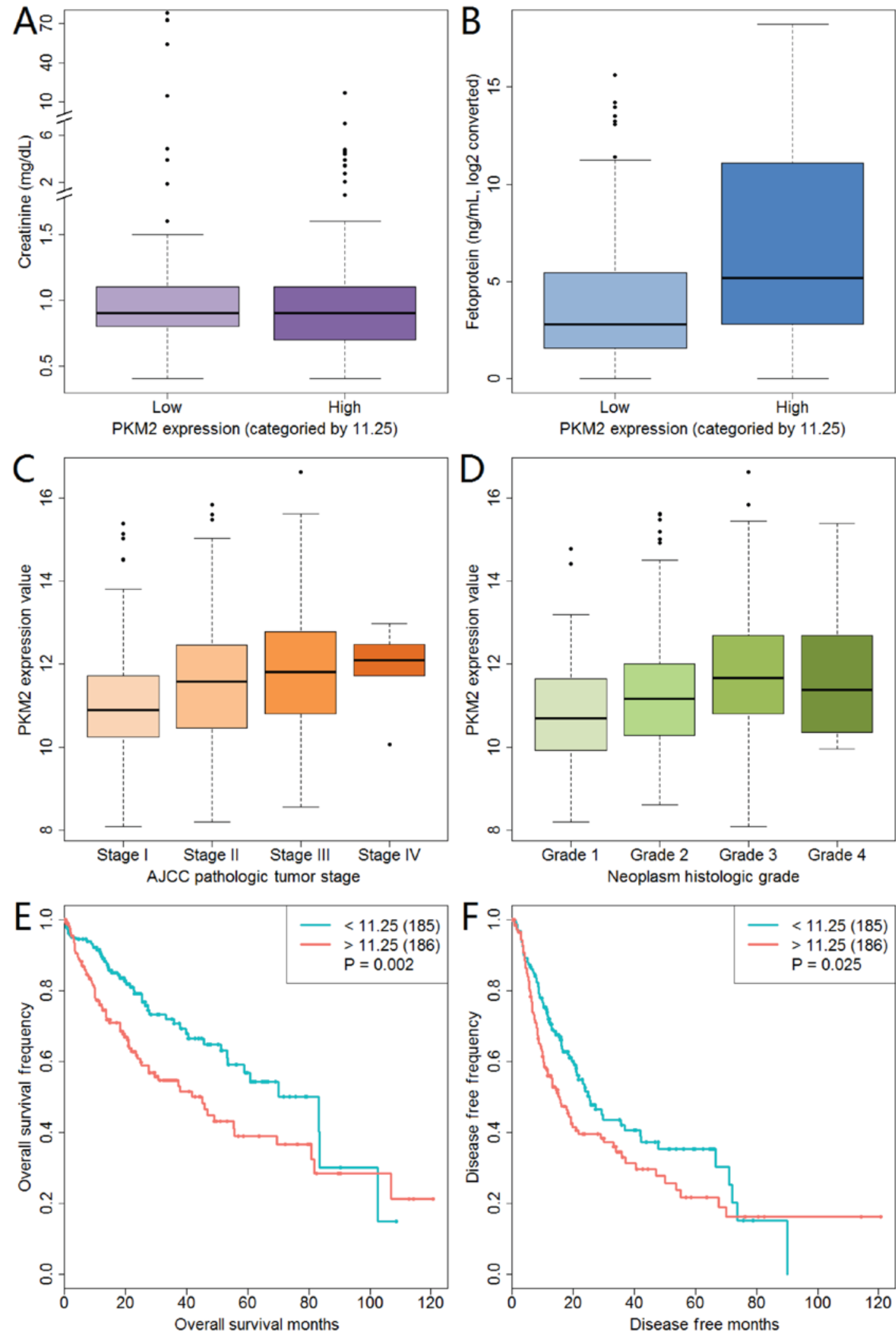

Figure 1. PKM2 expression and its association with clinicopathological characteristics. Correlation of PKM2 expression with (A) creatinine, (B) fetoprotein, (C) tumor stage and (D) histologic grade. (E) Effect of PKM2 expression on overall survival in liver cancer patients. (F) Effect of PKM2 expression on disease-free survival in liver cancer patients. PKM2, pyruvate kinase muscle isozyme M2; AJCC, American Joint Committee on Cancer.

efforts have been made to search for potential novel and effective molecular-targeted therapy. However, the underlying molecular mechanisms and biomarkers for the progression and metastasis of HCC remain largely unknown.
A previous study suggested that PKM2 might play a significant role in the aggressiveness and poor prognosis of HCC, and its expression may act as a biomarker for the prognosis of HCC $(27,28)$. In our previous study, we found 
Table II. Correlation between clinicopathologic characteristics and PKM2 expression ${ }^{\mathrm{a}}$.

\begin{tabular}{lrrr}
\hline Clinicopathological variables & Total & Odds ratio (95\% CI) & P-value \\
\hline Sex (male vs. female) & 370 & $2.407(1.537-3.771)$ & $<0.001$ \\
Diagnosis age (continuous) & 370 & $0.988(0.973-1.003)$ & 0.130 \\
BMI (continuous) & 334 & $0.985(0.951-1.020)$ & 0.394 \\
Creatinine (continuous) & 298 & $0.962(0.911-1.015)$ & 0.153 \\
Fetoprotein (continuous, log2 converted) & 277 & $1.157(1.091-1.228)$ & $<0.001$ \\
Albumin (continuous) & 295 & $0.961(0.899-1.026)$ & 0.233 \\
Residual tumor (R0 vs. R1 or R2) & 364 & $2.293(1.142-4.601)$ & 0.020 \\
Vascular tumor invasion (none vs. micro or macro) & 315 & $1.521(0.954-2.425)$ & 0.078 \\
Adjacent hepatic tissue inflammation (none vs. mild or severe) & 234 & $1.000(0.598-1.673)$ & $>0.999$ \\
Stage (I or II vs. III or IV) & 348 & $1.857(1.141-3.021)$ & 0.013 \\
Grade (1 or 2 vs. 3 or 4) & 366 & $2.010(1.303-3.100)$ & 0.002
\end{tabular}

${ }^{a}$ Categorical dependent variable, greater or less than the median expression level (11.25). PKM2, pyruvate kinase muscle isozyme M2; CI, confidence interval; BMI, body mass index.

Table III. Association between clinicopathological characteristics and overall survival.

\begin{tabular}{lcrr}
\hline Clinicopathologic variables & Total & Hazard ratio (95\% CI) & P-value \\
\hline PKM2 expression (continuous) & 371 & $1.192(1.075-1.321)$ & 0.001 \\
PKM2 expression (categorical, above or below median of 11.25) & 371 & $1.740(1.223-2.475)$ & 0.002 \\
Sex (male vs. female) & 370 & $1.225(0.860-1.746)$ & 0.260 \\
Diagnosis age (continuous) & 370 & $1.012(0.999-1.026)$ & 0.078 \\
BMI (continuous) & 334 & $0.971(0.939-1.004)$ & 0.086 \\
Creatinine (continuous) & 298 & $0.989(0.963-1.017)$ & 0.437 \\
Fetoprotein (continuous, log2 converted) & 277 & $1.036(0.992-1.083)$ & 0.107 \\
Albumin (continuous) & 295 & $0.986(0.943-1.032)$ & 0.550 \\
Residual tumor (R0 vs. R1 or R2) & 364 & $2.033(1.215-3.401)$ & 0.007 \\
Vascular tumor invasion (none vs. micro or macro) & 315 & $1.348(0.890-2.042)$ & 0.159 \\
Adjacent hepatic tissue inflammation (none vs. mild or severe) & 234 & $0.815(0.501-1.325)$ & 0.409 \\
Stage (I or II vs. III or IV) & 348 & $2.443(1.691-3.529)$ & $<0.001$ \\
Grade (1 or 2 vs. 3 or 4) & 366 & $1.120(0.781-1.606)$ & 0.539
\end{tabular}

PKM2, pyruvate kinase muscle isozyme M2; CI, confidence interval; BMI, body mass index.

that HCC patients with lower levels of PKM2 expression are more sensitive to radiotherapy. However, there was no significant correlation between PKM2 expression and clinicopathological features, such as sex, age, history of hepatitis, history of liver cirrhosis, alpha fetoprotein, aspartate aminotransferase, alanine aminotransferase and clinical stage (26). In the present study, the results showed that the high PKM2 expression group showed lower creatinine levels, higher fetoprotein levels and more severe cancer progression compared with the low PKM2 expression group. These differences in findings might be due to the relatively small number of cases in our previous study and that the patients were all Chinese. Therefore, these differences may be caused by sample size and differences ethnicities of the participants. In addition, a high PKM2 expression was associated with poor overall survival in HCC patients, which is independent of other clinicopathological characteristics.
In recent years, the over-expression of PKM2 has been observed in numerous cancers, including gastric (33), lung (34) and breast tumors $(35,36)$. Furthermore, PMK2 has been reported to function as a protein kinase and a transcriptional coactivator with the ability to regulate signaling pathways, cell cycle progression, gene expression and metabolism $(18,37,38)$. Moreover, recent findings showed that a high expression of PKM2 appears in a multitude of inflammatory diseases, and PKM2 has been reported as a novel potential molecular target in anti-inflammatory therapies (25). Additionally, a meta-analysis has been demonstrated to reveal a significant correlation between the over-expression of PKM2 and overall survival as well as clinical pathological features in solid tumors of the digestive cancers, thereby illustrating that PKM2 might be employed as an indicator for metastasis and poor prognosis in cancers of the digestive system (39). All of these findings suggest the central role of PKM2 in tumorigenesis. 
Table IV. Association between clinicopathologic characteristics and disease free survival.

\begin{tabular}{|c|c|c|c|}
\hline Clinicopathological variables & Total & Hazard ratio $(95 \% \mathrm{CI})$ & P-value \\
\hline PKM2 expression (continuous) & 371 & $1.129(1.023-1.247)$ & 0.016 \\
\hline PKM2 expression (categorical, above or below median of 11.25) & 371 & $1.406(1.044-1.895)$ & 0.025 \\
\hline Sex (male vs. female) & 370 & $1.155(0.842-1.583)$ & 0.372 \\
\hline Diagnosis age (continuous) & 370 & $0.997(0.985-1.009)$ & 0.625 \\
\hline BMI (continuous) & 334 & $0.984(0.958-1.010)$ & 0.220 \\
\hline Creatinine (continuous) & 298 & $1.002(0.987-1.017)$ & 0.819 \\
\hline Fetoprotein (continuous, $\log 2$ converted) & 277 & $1.038(1.001-1.077)$ & 0.047 \\
\hline Albumin (continuous) & 295 & $1.009(0.983-1.036)$ & 0.507 \\
\hline Residual tumor (R0 vs. R1 or R2) & 364 & $1.763(1.091-2.848)$ & 0.021 \\
\hline Vascular tumor invasion (none vs. micro or macro) & 315 & $1.990(1.407-2.814)$ & $<0.001$ \\
\hline Adjacent hepatic tissue inflammation (none vs. mild or severe) & 234 & $0.833(0.577-1.204)$ & 0.332 \\
\hline Stage (I or II vs. III or IV) & 348 & $2.376(1.712-3.298)$ & $<0.001$ \\
\hline Grade ( 1 or 2 vs. 3 or 4$)$ & 366 & $1.106(0.811-1.507)$ & 0.525 \\
\hline
\end{tabular}

CI, confidence interval; BMI, body mass index; PKM2, pyruvate kinase muscle isozyme M2.

Table V. Multivariate survival model after variable selection.

\begin{tabular}{lrr}
\hline Variables & Total & Hazard ratio (95\% CI) \\
\hline Overall survival $^{\mathrm{a}}$ & & \\
PKM2 expression (continuous) & 371 & $1.119(1.000-1.253)$ \\
PKM2 expression (categorical, above or below median of 11.25) & 371 & $1.477(1.014-2.151)$ \\
Disease free survival & & 0.050 \\
PKM2 expression (continuous) & 371 & $1.045(0.913-1.196)$ \\
PKM2 expression (categorical, above or below median of 11.25) & 371 & $1.194(0.799-1.785)$ \\
\hline
\end{tabular}

${ }^{a}$ Adjusted for residual tumor and stage. ${ }^{\mathrm{b}}$ Adjusted for fetoprotein, residual tumor, vascular tumor invasion and stage. CI, confidence interval; PKM2, pyruvate kinase muscle isozyme M2.

PKM2 not only play a dominant role in proliferation and cancer cell metabolism, but it is also abundantly expressed in HCC. Recent studies demonstrated that PKM2 plays a key role in the aggressiveness and poor prognosis of HCC. PKM2 expression could be a biomarker for the prognosis of $\mathrm{HCC}$ and a potential molecular target for the development of anti-cancer drugs (28). Furthermore, a recent study illuminated that a low expression of PKM2 inhibited the proliferation and migration of HCC cells, as well as angiogenesis and autophagy (29). A low PKM2 expression also induced apoptosis as shown by in vitro and in vivo assays, and immunohistochemical analysis involving $721 \mathrm{HCC}$ patients after curative resection was performed to analyze the poor prognosis of PKM2 (29). However, a study involving $\mathrm{Pkm} 2^{-/-}$mice has also shown that PKM2 is not necessary for postnatal or embryonic development due to compensatory mechanism of PKM isoforms and that a loss of PKM2 leads to the spontaneous development of HCC, which is inconsistent with a number of existing models on PKM2 function. These findings suggest that PKM2 plays a key role in controlling systemic energy and metabolic homeostasis as well as preventing spontaneous HCC (40). The previous study showed that the upregulation of PKM2 in primary and validation cohorts was linked to a high tumor-node metastasis (TNM) stage, which was consistent with our present study (28). In summary, our study suggested that PKM2 has been shown to play a dominant role in the overall survival and pathological damage of HCC.

In conclusion, combined with previous studies, we further confirmed that a high expression of PKM2 is strongly associated with poor overall survival in patients with HCC. In particular, we found that the effect of PKM2 on the prognosis of HCC patients is independent of clinicopathological features. Liver cancer is a malignant tumor with poor therapeutic effects. We hypothesized that investigating the correlation between PKM2 and $\mathrm{HCC}$, and further revealing the relationship between PKM2 and the sensitivity of HCC treatment may increase therapeutic efficacy and prolong patient survival. Therefore, PKM2 expression might be a novel prognostic indicator for HCC patients after curative resection, and this may be helpful for designing therapies that target PKM2.

There are several limitations in this study. Firstly, the results were not significant in the analysis of the association between PKM2 expression levels and disease-free survival with the adjustment of co-variables. Secondly, all the findings are based 
on the reanalysis of TCGA data, and protein data were not available to confirm these results. Finally, the data regarding drug treatment and prognosis of HCC were not available and therefore limit the analysis of clinical outcomes in this study. Given the limitations of our study, further in-depth studies that involve a larger number of samples are required to confirm these results.

\section{Acknowledgements}

Not applicable.

\section{Funding}

The present study was supported by Anhui Provincial Natural Science Foundation (grant no. 1808085MH239), the Instruments Function Deployment Foundation of CAS (grant nos. 2014g0019, 2016g0049 and 2017gz005) and the CAS Key Technology Talent Program.

\section{Availability of data and materials}

The datasets used and/or analyzed during the current study are available from the Genomic Data Commons Data Portal (portal.gdc.cancer.gov/repository). The files of miRNA Expression Quantification and Clinical Supplement in TCGA-LIHC were downloaded and analyzed in the present study.

\section{Authors' contributions}

DHL, WXL and YDG designed the study. WXL and WWL performed data collection. WXL, WWL and DHL conducted data analysis. All authors wrote the manuscript. WXL, DHL and YDG revised the manuscript. The final version of the manuscript has been read and approved by all authors.

\section{Ethics approval and consent to participate}

Not applicable.

\section{Patient consent for publication}

Not applicable.

\section{Competing interests}

The authors declare that they have no competing interests.

\section{References}

1. Kumar M, Zhao X and Wang XW: Molecular carcinogenesis of hepatocellular carcinoma and intrahepatic cholangiocarcinoma: One step closer to personalized medicine? Cell Biosci 1: 5, 2011.

2. Diaz-Gonzalez A, Forner A, Rodriguez de Lope C and Varela M: New challenges in clinical research on hepatocellular carcinoma. Rev Esp Enferm Dig 108: 485-493, 2016.

3. Allemani C, Weir HK, Carreira H, Harewood R, Spika D, Wang XS, Bannon F, Ahn JV, Johnson CJ, Bonaventure A, et al: Global surveillance of cancer survival 1995-2009: Analysis of individual data for 25676887 patients from 279 population-based registries in 67 countries (CONCORD-2). Lancet 385: 977-1010, 2015 .
4. Ferlay J, Soerjomataram I, Dikshit R, Eser S, Mathers C, Rebelo M, Parkin DM, Forman D and Bray F: Cancer incidence and mortality worldwide: Sources, methods and major patterns in GLOBOCAN 2012. Int J Cancer 136: E359-E386, 2015.

5. Venook AP, Papandreou C, Furuse J and de Guevara LL: The incidence and epidemiology of hepatocellular carcinoma: A global and regional perspective. Oncologist 15 (Suppl 4): S5-S13, 2010.

6. Chen W, Zheng R, Zeng H, Zhang S and He J: Annual report on status of cancer in China, 2011. Chin J Cancer Res 27: 2-12, 2015.

7. Liu M, Jiang L and Guan XY: The genetic and epigenetic alterations in human hepatocellular carcinoma: A recent update. Protein Cell 5: 673-691, 2014.

8. El-Serag HB: Hepatocellular carcinoma. N Engl J Med 365: 1118-1127, 2011.

9. Rasool M, Rashid S, Arooj M, Ansari SA, Khan KM, Malik A, Naseer MI, Zahid S, Manan A, Asif M, et al: New possibilities in hepatocellular carcinoma treatment. Anticancer Res 34: 1563-1571, 2014

10. Bruix J, Gores GJ and Mazzaferro V: Hepatocellular carcinoma: Clinical frontiers and perspectives. Gut 63: 844-855, 2014.

11. Park US, Park SK, Lee YI, Park JG and Lee YI: Hepatitis B virus-X protein upregulates the expression of $\mathrm{p} 21 \mathrm{waf} 1 / \mathrm{cip} 1$ and prolongs G1->S transition via a p53-independent pathway in human hepatoma cells. Oncogene 19: 3384-3394, 2000.

12. Arbuthnot P, Capovilla A and Kew M: Putative role of hepatitis $\mathrm{B}$ virus $\mathrm{X}$ protein in hepatocarcinogenesis: Effects on apoptosis, DNA repair, mitogen-activated protein kinase and JAK/STAT pathways. J Gastroenterol Hepatol 15: 357-368, 2000.

13. Christofk HR, Vander Heiden MG, Harris MH, Ramanathan A, Gerszten RE, Wei R, Fleming MD, Schreiber SL and Cantley LC: The M2 splice isoform of pyruvate kinase is important for cancer metabolism and tumour growth. Nature 452: 230-233, 2008.

14. Aravalli RN, Steer CJ and Cressman EN: Molecular mechanisms of hepatocellular carcinoma. Hepatology 48: 2047-2063, 2008.

15. Macintyre AN and Rathmell JC: PKM2 and the tricky balance of growth and energy in cancer. Mol Cell 42: 713-714, 2011.

16. Garnett ME, Dyson RD and Dost FN: Pyruvate kinase isozyme changes in parenchymal cells of regenerating rat liver. J Biol Chem 249: 5222-5226, 1974.

17. Lu Z: Nonmetabolic functions of pyruvate kinase isoform M2 in controlling cell cycle progression and tumorigenesis. Chin $\mathrm{J}$ Cancer 31: 5-7, 2012.

18. Yang W, Xia Y, Hawke D, Li X, Liang J, Xing D, Aldape K, Hunter T, Alfred Yung WK and Lu Z: PKM2 phosphorylates histone $\mathrm{H} 3$ and promotes gene transcription and tumorigenesis. Cell 150: 685-696, 2012.

19. Wong CC, Au SL, Tse AP, Xu IM, Lai RK, Chiu DK, Wei LL, Fan DN, Tsang FH, Lo RC, et al: Switching of pyruvate kinase isoform $\mathrm{L}$ to $\mathrm{M} 2$ promotes metabolic reprogramming in hepatocarcinogenesis. PLoS One 9: e115036, 2014.

20. Peng XC, Gong FM, Zhao YW, Zhou LX, Xie YW, Liao HL, Lin HJ, Li ZY, Tang MH and Tong AP: Comparative proteomic approach identifies PKM2 and cofilin-1 as potential diagnostic, prognostic and therapeutic targets for pulmonary adenocarcinoma. PLoS One 6: e27309, 2011.

21. Kumar Y, Tapuria N, Kirmani N and Davidson BR: Tumour M2-pyruvate kinase: A gastrointestinal cancer marker. Eur J Gastroenterol Hepatol 19: 265-276, 2007.

22. Landt S, Jeschke S, Koeninger A, Thomas A, Heusner T, Korlach S, Ulm K, Schmidt P, Blohmer JU, Lichtenegger W, et al: Tumor-specific correlation of tumor M2 pyruvate kinase in pre-invasive, invasive and recurrent cervical cancer. Anticancer Res 30: 375-381, 2010.

23. Haug U, Hundt S and Brenner H: Sensitivity and specificity of faecal tumour M2 pyruvate kinase for detection of colorectal adenomas in a large screening study. Br J Cancer 99: 133-155, 2008.

24. Zhu H, Luo H, Zhu X, Hu X, Zheng L and Zhu X: Pyruvate kinase M2 (PKM2) expression correlates with prognosis in solid cancers: A meta-analysis. Oncotarget 8: 1628-1640, 2017.

25. Alves-Filho JC and Palsson-McDermott EM: Pyruvate Kinase M2: A potential target for regulating inflammation. Front Immunol 7: 145, 2016.

26. Lu DH, Yu HQ, Wang Q, Liu JT and Sun GP: Expression of pyruvate kinase M2 in hepatocellular carcinoma and its clinical significance. Anhui Med Pharma 20: 707-710, 2016 (In Chinese).

27. Liu Y, Wu H, Mei Y, Ding X, Yang X, Li C, Deng M and Gong J: Clinicopathological and prognostic significance of PKM2 protein expression in cirrhotic hepatocellular carcinoma and non-cirrhotic hepatocellular carcinoma. Sci Rep 7: 15294, 2017. 
28. Chen Z, Lu X, Wang Z, Jin G, Wang Q, Chen D, Chen T, Li J, Fan J, Cong W, et al: Co-expression of PKM2 and TRIM35 predicts survival and recurrence in hepatocellular carcinoma. Oncotarget 6: 2538-2548, 2015.

29. Liu WR, Tian MX, Yang LX, Lin YL, Jin L, Ding ZB, Shen YH, Peng YF, Gao DM, Zhou J, et al: PKM2 promotes metastasis by recruiting myeloid-derived suppressor cells and indicates poor prognosis for hepatocellular carcinoma. Oncotarget 6: 846-861, 2015.

30. Gao J, Aksoy BA, Dogrusoz U, Dresdner G, Gross B, Sumer SO, Sun Y, Jacobsen A, Sinha R, Larsson E, et al: Integrative analysis of complex cancer genomics and clinical profiles using the cBioPortal. Sci Signal 6: pl1, 2013.

31. Eggert T, McGlynn KA, Duffy A, Manns MP, Greten TF and Altekruse SF: Epidemiology of fibrolamellar hepatocellular carcinoma in the USA, 2000-10. Gut 62: 1667-1668, 2013

32. Mittal S and El-Serag HB: Epidemiology of hepatocellular carcinoma: Consider the population. J Clin Gastroenterol 47 (Suppl): S2-S6, 2013.

33. Kefas B, Comeau L, Erdle N, Montgomery E, Amos S and Purow B: Pyruvate kinase M2 is a target of the tumor-suppressive microRNA-326 and regulates the survival of glioma cells. Neuro Oncol 12: 1102-1112, 2010.

34. Shi HS, Li D, Zhang J, Wang YS, Yang L, Zhang HL, Wang XH, Mu B, Wang W, Ma Y, et al: Silencing of pkm2 increases the efficacy of docetaxel in human lung cancer xenografts in mice. Cancer Sci 101: 1447-1453, 2010.

35. Israelsen WJ, Dayton TL, Davidson SM, Fiske BP, Hosios AM Bellinger G, Li J, Yu Y, Sasaki M, Horner JW, et al: PKM2 isoform-specific deletion reveals a differential requirement for pyruvate kinase in tumor cells. Cell 155 397-409, 2013
36. Wang $\mathrm{YH}$, Israelsen WJ, Lee $\mathrm{D}, \mathrm{Yu} \mathrm{VW}$, Jeanson $\mathrm{NT}$, Clish CB, Cantley LC, Vander Heiden MG and Scadden DT: Cell-state-specific metabolic dependency in hematopoiesis and leukemogenesis. Cell 158: 1309-1323, 2014.

37. Yang W, Xia Y, Ji H, Zheng Y, Liang J,Huang W, Gao X, Aldape K and $\mathrm{Lu} \mathrm{Z}$ : Nuclear PKM2 regulates $\beta$-catenin transactivation upon EGFR activation. Nature 480: 118-122, 2011.

38. Zhao X, Zhao L, Yang H, Li J, Min X, Yang F, Liu J and Huang G: Pyruvate kinase M2 interacts with nuclear sterol regulatory element-binding protein 1a and thereby activates lipogenesis and cell proliferation in hepatocellular carcinoma. J Biol Chem 293: 6623-6634, 2018.

39. Wu J, Hu L, Chen M, Cao W, Chen $\mathrm{H}$ and He T: Pyruvate kinase M2 overexpression and poor prognosis in solid tumors of digestive system: evidence from 16 cohort studies. Onco Targets Ther 9: 4277-4288, 2016.

40. Dayton TL, Gocheva V, Miller KM, Israelsen WJ, Bhutkar A, Clish CB, Davidson SM, Luengo A, Bronson RT, Jacks $\mathrm{T}$, et al: Germline loss of PKM2 promotes metabolic distress and hepatocellular carcinoma. Genes Dev 30: 1020-1033, 2016. International (CC BY-NC-ND 4.0) License. 